\title{
SELF-COMMUTATOR NORM OF HYPONORMAL TOEPLITZ OPERATORS
}

\author{
TRIEU LE
}

Abstract. Chu and Khavinson recently obtained a lower bound for the norm of the self-commutator of a certain class of hyponormal Toeplitz operators on the Hardy space. Via a different approach, we offer a generalization of their result.

Mathematics subject classification (2010): Primary 47B35.

Keywords and phrases: Toeplitz operator, Hardy space, hyponormality, spectral area.

\section{REFERENCES}

[1] S. R. Bell, T. Ferguson, And E. Lundberg, Self-commutators of Toeplitz operators and isoperimetric inequalities, Math. Proc. R. Ir. Acad. 114A (2014), no. 2, 115-133. MR 3353499

[2] C. BÉnÉtEAU AND D. KHAVINSON, The isoperimetric inequality via approximation theory and free boundary problems, Comput. Methods Funct. Theory 6 (2006), no. 2, 253-274. MR 2291136

[3] A. BRown And P. R. Halmos, Algebraic properties of Toeplitz operators, J. Reine Angew. Math. 213 (1963/1964), 89-102. MR 0160136 (28 \#3350)

[4] C. Chu And D. Khavinson, A note on the spectral area of Toeplitz operators, Proc. Amer. Math. Soc. 144 (2016), no. 6, 2533-2537. MR 3477069

[5] C. C. Cowen, Hyponormality of Toeplitz operators, Proc. Amer. Math. Soc. 103 (1988), no. 3, 809812. MR 947663

[6] M. FleEman And D. Khavinson, Extremal domains for self-commutators in the Bergman space, Complex Anal. Oper. Theory 9 (2015), no. 1, 99-111. MR 3300527

[7] D. Khavinson, A note on Toeplitz operators, Banach spaces (Columbia, Mo., 1984), Lecture Notes in Math., vol. 1166, Springer, Berlin, 1985, pp. 89-94. MR 827763

[8] J. Olsen AND M. C. REguera, On a sharp estimate for Hankel operators and Putnam's inequality, Rev. Mat. Iberoam. 32 (2016), no. 2, 495-510. MR 3512424

[9] C. R. Putnam, An inequality for the area of hyponormal spectra, Math. Z. 116 (1970), 323-330. MR 0270193 